\title{
The European Sleep Apnoea Database (ESADA): report from 22 European sleep laboratories
}

\author{
J. Hedner, L. Grote, M. Bonsignore, W. McNicholas, P. Lavie, G. Parati, P. Sliwinski, \\ F. Barbé, W. De Backer, P. Escourrou, I. Fietze, J.A. Kvamme, C. Lombardi, \\ O. Marrone, J.F. Masa, J.M. Montserrat, T. Penzel, M. Pretl, R. Riha, D. Rodenstein, \\ T. Saaresranta, R. Schulz, R. Tkacova, G. Varoneckas, A. Vitols, \\ H. Vrints and J. Zielinski
}

ABSTRACT: The European Sleep Apnoea Database (ESADA) reflects a network of 22 sleep disorder centres in Europe enabled by a COST action B26 programme. This ongoing project aims to describe differences in standard clinical care of patients with obstructive sleep apnoea (OSA) and to establish a resource for genetic research in this disorder.

Patients with suspected OSA are consecutively included and followed up according to local clinical standards. Anthropometrics, medical history, medication, daytime symptoms and sleep data (polysomnography or cardiorespiratory polygraphy) are recorded in a structured web-based report form.

5,103 patients $(1,426$ females, mean \pm SD age $51.8 \pm 12.6 \mathrm{yrs}, 79.4 \%$ with apnoea/hypopnoea index $(A H I) \geqslant 5$ events $\cdot h^{-1}$ ) were included from March 15, 2007 to August 1, 2009. Morbid obesity (body mass index $\geqslant 35 \mathrm{~kg} \cdot \mathrm{m}^{-2}$ ) was present in $21.1 \%$ of males and $28.6 \%$ of females. Cardiovascular, metabolic and pulmonary comorbidities were frequent $(49.1 \%, 32.9 \%$ and $14.2 \%$, respectively). Patients investigated with a polygraphic method had a lower $\mathrm{AHI}$ than those undergoing polysomnography (23.2 \pm 23.5 versus $29.1 \pm 26.3$ events $\left.\cdot h^{-1}, p<0.0001\right)$.

The ESADA is a rapidly growing multicentre patient cohort that enables unique outcome research opportunities and genotyping. The first cross-sectional analysis reveals a high prevalence of cardiovascular and metabolic morbidity in patients investigated for OSA.

KEYWORDS: Comorbidity, European Sleep Apnoea Database, genetic, multicentre cohort, obstructive sleep apnoea syndrome

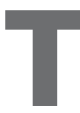

he obstructive sleep apnoea (OSA) syndrome is a highly prevalent disorder, affecting at least $4 \%$ of adult males and $2 \%$ of adult females [1, 2]. The disorder is associated with recurring episodes of partial or complete upper airway occlusion during sleep, which results in marked sleep disturbance, excessive daytime sleepiness, irritability and morning headache, among other symptoms. Sleep apnoea is strongly associated with impaired neuropsychological function and reduced quality of life and social functioning [3]. Daytime sleepiness leads to an increased risk for traffic and industrial accidents [4]. OSA is also independently associated with several cardiovascular complications: not only systemic hypertension but also ischaemic heart disease and stroke [5-7].

A collaborative European network was established in 2005, as part of the European Union Cooperation in Science and Technology (COST)
Action B26 programme, for the purpose of investigating the impact of OSA as a public health burden in Europe. The primary objectives of COST Action B26 were to assess the role of OSA as a possible cause of increased cardiovascular risk and to coordinate studies on pathogenetic mechanisms of increased cardiovascular risk associated with exposure to intermittent hypoxia. Secondary objectives were to promote exchanges between European research groups on the following topics: 1) diagnosis of OSA and patient management; 2) excessive daytime sleepiness and medico-legal implications of OSA; 3) a common protocol to be adopted by all participating centres for data collection during clinical follow-up of treated and untreated OSA patients representative of the European population; and 4) genetic mechanisms of OSA with particular reference to cardiovascular disease, obesity and metabolic syndrome.
AFFILIATIONS

For a full list of author affiliations, see the Acknowledgements section.

\section{CORRESPONDENCE}

L. Grote

ESADA office, Gothenburg University Centre for Sleep and Vigilance

Disorders

Medicinaregatan 8B Box 421

S-405 30 Gothenburg

Sweden

E-mail: ludger.grote@lungall.gu.se

Received:

March 242010

Accepted after revision:

Jan 282011

First published online:

May 262011 
In the framework of COST B26 activities, a collaborative project was initiated, named the European Sleep Apnoea Database (ESADA), with the objective of recruiting a large prospective cohort of patients with suspected OSA. The current study deals with a number of questions addressed by the ESADA project. Since the project includes patient data from 22 centres throughout Europe and Israel, the database allows assessment of clinical practices in different countries relating to OSA and investigation of how differences in local practices may influence diagnosis and treatment decisions. The data also allow an assessment of different patient profiles among European sleep centres and exploration of whether previously described relationships between sleep apnoea and anthropometrics/comorbidities still pertain despite the different clinical/diagnostic procedures used in different European countries.

The current study addresses the methodological details of the ESADA project, including the project design and the techniques employed for data collection across participating centres. The cross-sectional data collected also provide the basis for longitudinal follow-up of the patient cohort.

\section{METHODS}

\section{General design of the ESADA}

The project aims to generate a multinational European database containing data from patients referred to sleep centres due to suspected OSA. In order to obtain a full cross-sectional sample, patients are included irrespective of comorbidity, concomitant medication and degree of sleepiness. Participating centres individually determine the proportion of locally referred patients to be included in the database, but each centre is encouraged to enrol as many patients as possible and no specific selection criteria have been applied to those to be enrolled. Thus, centres are requested not to limit recruitment to factors such as clinician on duty, clinics addressing specific patient groups or certain weekdays. These measures aim to limit unintended bias in the recruitment process. Moreover, each centre employs its own established clinical and diagnostic procedures with no attempt to enforce conformity on this process. Data on anthropometrics, clinical and laboratory findings as well as questionnaire-based data are transferred and stored in a central database based at the University of Gothenburg, Gothenburg, Sweden. A joint scientific committee was formed, comprising representatives from the different participating centres, to supervise the data collection and analysis, to resolve any practical issues related to the study as well as to coordinate and direct all scientific issues related to the ongoing projects.

\section{Study centres}

Study centres were recruited from those represented by the participating members of the COST Action B26. In total, 22 centres (20 of which are university-affiliated sleep clinics) representing 16 countries are participating. The affiliations of the centres include pulmonary medicine, neurology and standalone units. All centres receive referrals from hospital specialists as well as from general practitioners. The clinical evaluation procedure includes an interview by a sleep physician followed by either an attended/unattended overnight polysomnography (PSG) $(n=1,602)$ or polygraphic study $(n=3,258)$, according to local practice but performed according to internationally accepted guidelines. Sleep records are scored at each of the centres based on standardised criteria.

\section{Inclusion and exclusion data}

Patients with suspected OSA (male or female, aged 18-80 yrs) are eligible for inclusion in the study. Patients must be able to speak, read and understand the local language and possess the ability to respond to questions and follow instructions. Exclusion criteria include patients with already treated sleep apnoea, a limited life expectancy due to illness unrelated to sleep apnoea (e.g. HIV, advanced renal disease, uncontrolled malignancies) or documented alcohol or drug abuse up to $1 \mathrm{yr}$ prior to inclusion in the study.

\section{Ethical considerations}

The agreed basic protocol was locally approved by the appropriate ethics committee at each of the centres. Future protocols added will be subject to ethical consideration according to local regulations. Wherever appropriate, patients are requested to provide written informed consent for participation in the database.

\section{Web-based data collection format and data quality assurance}

The ESADA employs a specifically designed web-based data collection format constructed for transfer of data to the central database at the University of Gothenburg. Each centre is equipped with a unique log-in to a uniform clinical report format (CRF) module containing six predefined submodules (all in the English language) for recording of data. The patient registry is coded and the identity of the patient is kept at the reporting centre and secured with a written patient identity log. Each centre has full access to data stored on its own patients. In order to ensure uniform data entry procedures and data quality, each centre has appointed a specific data manager who was individually instructed by a central study monitor from Gothenburg regarding the type of data in the database, entry procedures and other quality control measures. This has been followed by a practical training session for the study monitor in each of the centres. A detailed protocol describing methods for data acquisition, calculation and reporting has been distributed to all participating centres. The quality assurance programme also includes detailed instructions on sleep study scoring rules, classification of comorbidities and coding of concomitant medication. Data quality and completeness is continuously checked by a study monitor with full access to the complete database. The ESADA web report format also enables new and specific CRF modules for specific sub-studies (e.g. specific patient groups, interventional trials, health economy evaluations) that may be initiated by participating centres within the network.

\section{Study flow}

Each new patient is evaluated at baseline and, if applicable, at clinically relevant follow-up visits (fig. 1). Separate CRF modules are provided for each new visit. A CRF page addresses a specific part of the clinical work-up. The protocol does not state a fixed time to follow-up but follows the clinical routine currently applied at each participating centre. 


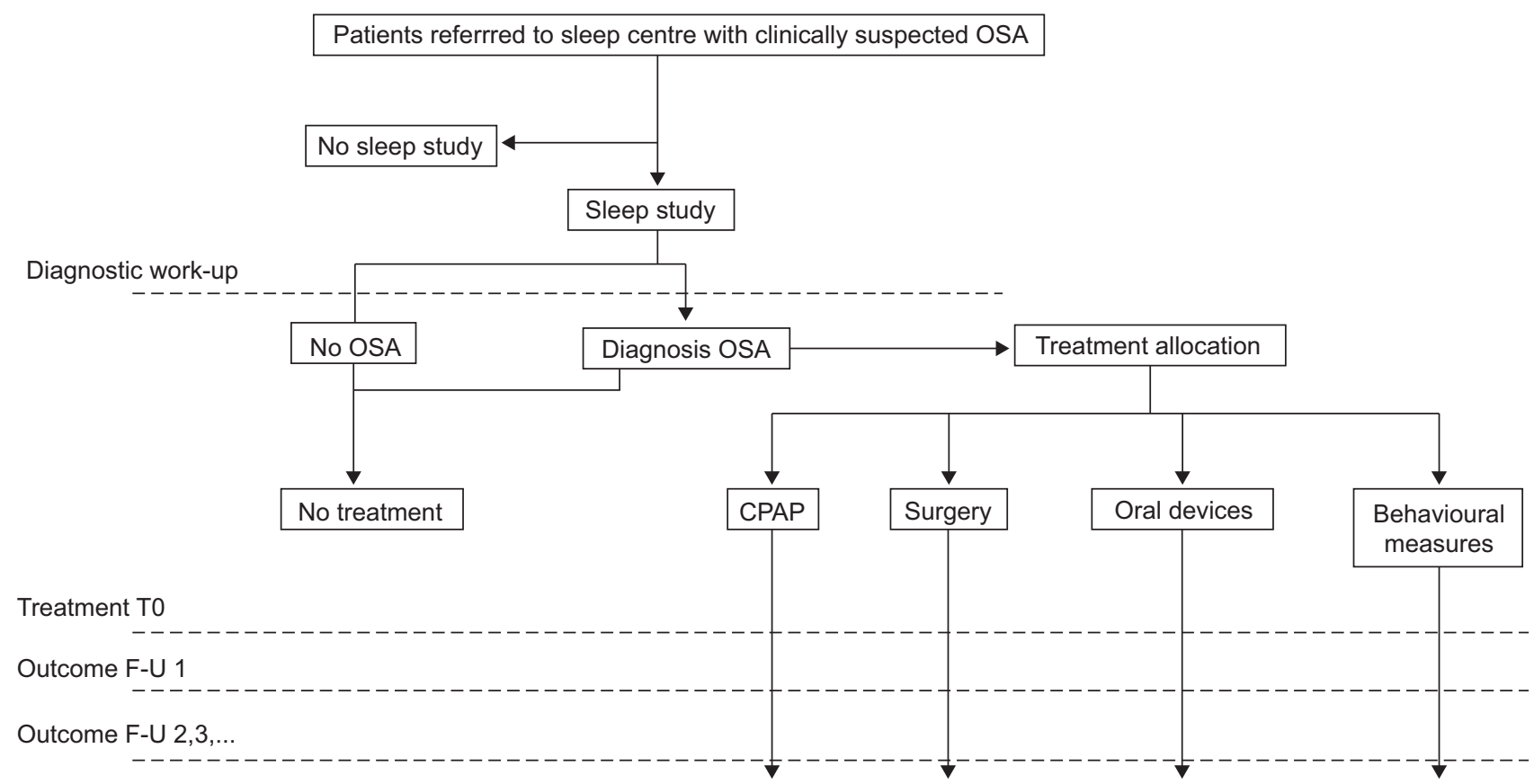

FIGURE 1. Overview of the European Sleep Apnoea Database (ESADA) study patient flow. OSA: obstructive sleep apnoea; CPAP: continuous positive airway pressure; F-U: follow-up

\section{Database structure}

Anthropometric data, medical history and concomitant medication A set of mandatory variables for each entry includes age at diagnosis, sex, race, height, weight, circumference of the neck, waist and hip, heart rate, and systolic and diastolic blood pressure (in a sitting position). A medical history including cardiovascular (e.g. hypertension, ischaemic heart disease, cerebrovascular disease), metabolic (e.g. diabetes, hyperlipidaemia, thyroid disease), pulmonary and psychiatric diseases as well as sleep disorders and other chronic diseases is collected for each patient. Information on all ongoing medications is obtained and reported individually according to the Anatomical Therapeutic Chemical (ATC) Classification System [8] for drug used.

\section{Sleep data}

The database has been constructed to accept either cardiorespiratory polygraphic or PSG data depending on local practice. The protocol states that limited-channel polygraphic recordings including a minimum of four channels (level 3 devices according to the American Association of Sleep Medicine (AASM)) [9]. All sleep data are visually examined and manually edited according to protocol definitions before entry into the database. Scoring rules applied according to the protocol are those proposed by the AASM in 2007 [10]. Thus, in a polygraphic recording, an apnoea/hypopnoea event may be scored if there is a clear decrease $(\geqslant 50 \%)$ in the amplitude of a valid measure of airflow (either by thermistors or nasal cannula pressure transducer) during sleep (in case of hypopnoea $\geqslant 3 \%$ oxygen desaturation is required) or the combination of a $\geqslant 30 \%$ reduction in airflow (compared with pre-event baseline) with $\geqslant 4 \%$ reduction of oxygen saturation. A minimum event duration of $10 \mathrm{~s}$ is required. In a PSG study, in addition to the criteria above, an event (with $\geqslant 50 \%$ flow reduction) that is associated with arousal is also classified as hypopnoea.
No specific distinction between apnoea and hypopnoea events is made in the report page. Data referring to calculation of indices are based on actual sleep time (PSG) or on time lapsed between lights off and lights on (limited-channel polygraphic recordings). Polygraphic recordings are requested to include at minimum the following information and indices: analysed time, subjective sleep time, apnoea/hypopnoea index (AHI), oxygen desaturation index (ODI-4\%), and mean and lowest arterial oxygen saturation. The following indices are reported in PSG recordings in addition to polygraphic recordings: total sleep time, sleep efficiency, percentage of different sleep stages, periodic limb movement (PLM) index, PLM arousal index, respiratory-related arousal index and spontaneous arousal index. In the PSG-based recordings, respiratory disturbance index is additionally calculated as AHI plus respiratory-related arousal index. Each recording is graded in terms of technical quality on a three-level rating scale. A separate section in the database includes information on follow-up studies.

\section{Blood tests}

The protocol states an option to include sampling of blood for analysis of total cholesterol, triglycerides, C-reactive protein, creatinine/urine albumin (optional) and HbA1c. Added protocols include specific blood samples to test various research hypotheses including genetic analysis. Blood samples for genetic analysis are kept under coded conditions at the discretion of each participating centre.

\section{Questionnaire data}

A variety of questionnaire-based instruments are administered at the different centres according to local practice. The following information is used for the purpose of the ESADA database. Sleep habit data are based on one question on usual sleep length and one question on usual sleep latency, and on 
results from the Epworth Sleepiness Scale (ESS) [11]. Data on health-related lifestyle and habits include alcohol consumption (units week $^{-1}$ ) and smoking (yes/no). Severity of disease is assessed by a Clinical Global Impression rating scale. This is a seven-point scale that requires the clinician to rate the severity of the patient's illness (sleep-disordered breathing) at the time of assessment relative to the clinician's experience. Information about driving licence (yes/no, according to European standard classification $\mathrm{A} / \mathrm{B}$ versus $\mathrm{C} / \mathrm{D} / \mathrm{E}$ ) and estimated distance of driving $\left(\mathrm{km} \cdot \mathrm{yr}^{-1}\right)$ is obtained.

\section{Statistics}

Continuous data were presented as mean \pm SD and compared by independent t-test. Ordinal data were presented as median and interquartile range and compared by Mann-Whitney U-test (SPSS version 17.0; SPSS, Inc., Chicago, IL, USA). A p-value $<0.01$ was considered statistically significant.

\section{RESULTS}

A total of 22 sleep laboratories currently participate in the ESADA project. Together, the centres have examined 5,103 patients, 3,677 males and 1,426 females (27.8\%), with suspected OSA from March 15, 2007 to August 1, 2009, and the recruitment rate in the study has increased to $\sim 200$ new cases per month. The number of recruited patients per centre ranged from 18 to 856. A blood sample for genetic analysis has been obtained from
$744(14.6 \%)$ patients. The incompleteness of various items in the database corresponds to $0.5-10 \%$. Sleep recording quality assessment ranged from excellent $(88.3 \%)$ to $1-2$ channels missing $(10.4 \%)$ or $3-4$ channels missing/poor quality $(1.3 \%)$.

The mean age of the analyses cohort was 51.8 yrs (median 53.0 yrs, 95\% CI 51.5-52.2 yrs) and the mean body mass index (BMI) was $31.1 \mathrm{~kg} \cdot \mathrm{m}^{-2}$ (median $30.1 \mathrm{~kg} \cdot \mathrm{m}^{-2}$, 95\% CI 31.0 $31.3 \mathrm{~kg} \cdot \mathrm{m}^{-2}$ ). Male patients were slightly younger and slightly less overweight compared with females (table 1). The distribution of obesity differed slightly between sexes, with more females in the normal as well as the morbidly obese range groups when compared with males $(20.1 \%$ versus $12.4 \%$ and $28.6 \%$ versus $21.1 \%$, respectively). Alcohol consumption was higher and smoking history was more frequent in male patients.

Daytime sleepiness was moderate, with a median ESS score of 10.0 [8]. Subjectively reported sleepiness was identical in males (median ESS 10.0 [8], $n=3,359$, ESS $>10$ in $43.8 \%$ ) and in females (median ESS 10.0 [8], $n=1,315$, ESS $>10$ in 46.5\%).

Sleep apnoea $(\mathrm{AHI} \geqslant 5)$ was diagnosed in $79.4 \%$ of included patients $(83.6 \%$ of males, $n=3,300$, and $68.5 \%$ of females, $\mathrm{n}=1,275)$ and the average severity was substantially higher in males compared with females (AHI $27.4 \pm 24.7$ and $18.3 \pm$ 22.4 events $\cdot \mathrm{h}^{-1}$, respectively; $\left.\mathrm{p}<0.001\right)$. The proportion of patients with severe sleep apnoea $\left(\geqslant 30\right.$ events $\left.\cdot h^{-1}\right)$ is shown in

TABLE 1 Selected anthropometric data, modifiable risk factors and comorbid conditions

\begin{tabular}{|c|c|c|c|}
\hline Age yrs & $53.6 \pm 12.6(1426)$ & $51.2 \pm 12.6(3677)$ & $51.8 \pm 12.6(5103)$ \\
\hline \multicolumn{4}{|l|}{ BMI } \\
\hline $\mathrm{kg} \cdot \mathrm{m}^{-2}$ & $31.5 \pm 7.6(1420)$ & $31.0 \pm 6.1(3658)$ & $31.1 \pm 6.5(5078)$ \\
\hline Normal, $<25 \mathrm{~kg} \cdot \mathrm{m}^{-2}$ & 20.1 & 12.4 & 14.5 \\
\hline Overweight, $25-<30 \mathrm{~kg} \cdot \mathrm{m}^{-2}$ & 28.1 & 37.5 & 34.8 \\
\hline Waist $\mathbf{c m}$ & $102.3 \pm 17.0(1399)$ & $108.5 \pm 15.0(3505)$ & $106.8 \pm 15.9(4904)$ \\
\hline Hip $\mathbf{c m}$ & $109.7 \pm 19.6(1399)$ & $107.6 \pm 14.2(3498)$ & $108.2 \pm 16.0(4897)$ \\
\hline Neck cm & $37.5 \pm 5.0(1404)$ & $42.5 \pm 4.1(3520)$ & $41.0 \pm 4.9(4924)$ \\
\hline Waist/hip ratio & $0.96 \pm 0.23(1399)$ & $1.02 \pm 0.16(3497)$ & $1.00 \pm 0.19(4896)$ \\
\hline Current smoker & 296/1388 (21.3) & $936 / 3596(26.0)$ & 1232/4984 (24.7) \\
\hline Alcohol consumption units week $^{-1}$ & $1.9 \pm 3.7(971)$ & $4.7 \pm 7.8(2726)$ & $4.0 \pm 7.1(3697)$ \\
\hline 1 & $491(35.7)$ & $1217(34.4)$ & $1708(34.7)$ \\
\hline 2 & $129(9.4)$ & $384(10.8)$ & $513(10.4)$ \\
\hline 3 & $30(2.2)$ & $110(3.1)$ & $140(2.8)$ \\
\hline$\geqslant 4$ & $20(1.4)$ & $36(1.1)$ & $56(1.1)$ \\
\hline \multicolumn{4}{|l|}{ Metabolic disease } \\
\hline Total & 487/1389 (35.0) & $1145 / 3575(32.1)$ & $1632 / 4964(32.9)$ \\
\hline \multicolumn{4}{|l|}{ Number of diagnoses } \\
\hline 1 & $370(26.6)$ & $835(23.4)$ & $1205(24.3)$ \\
\hline 2 & $93(6.7)$ & $260(7.3)$ & $353(7.1)$ \\
\hline$\geqslant 3$ & $24(1.7)$ & $50(1.4)$ & $72(1.5)$ \\
\hline
\end{tabular}




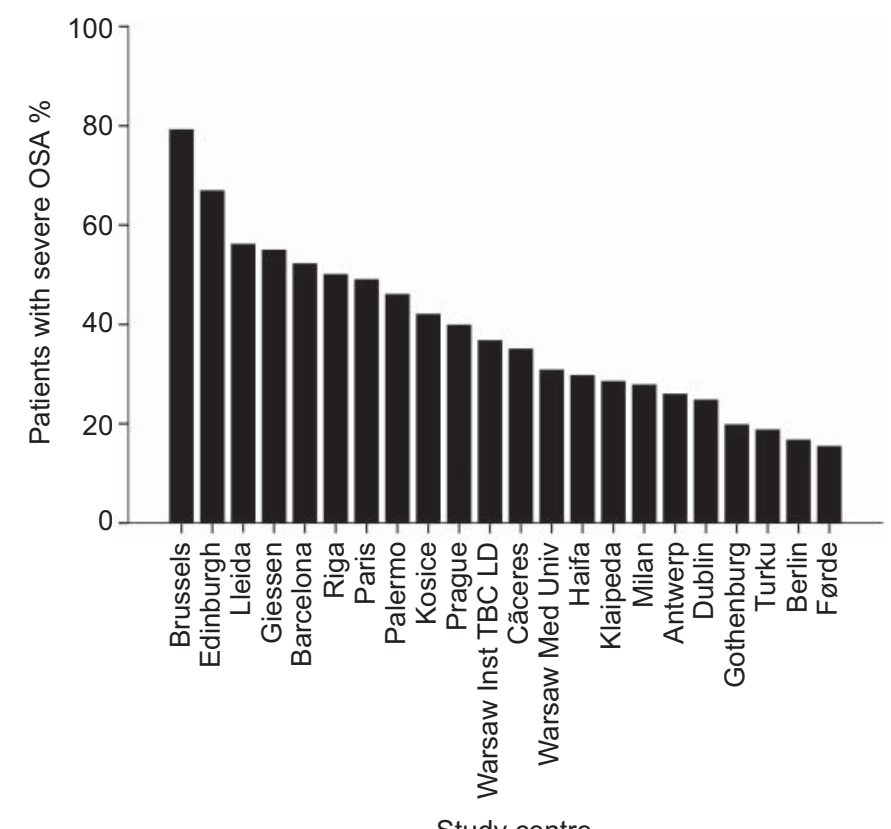

Study centre

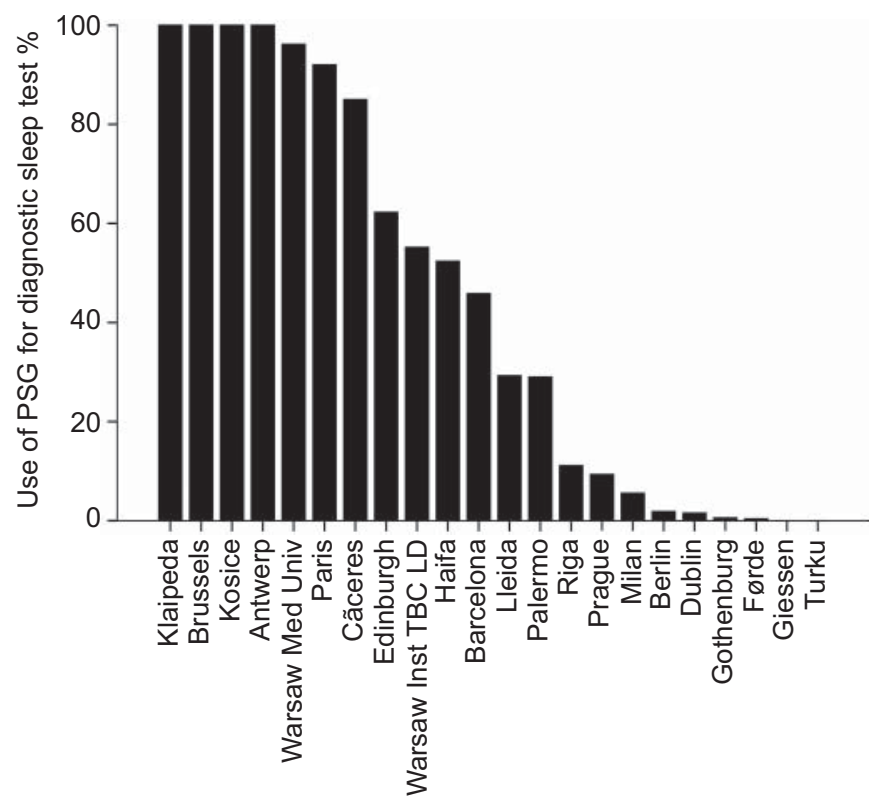

Study centre
FIGURE 2. The proportion of patients with severe obstructive sleep apnoea (OSA) (apnoea/hypopnoea index $\geqslant 30$ events $\cdot h^{-1}$ ) at each centre participating in the European Sleep Apnoea Database (ESADA) study. Inst TBC LD: Institute of Tuberculosis and Lung Diseases; Med Univ: Medical University.

figure 2. The method for diagnosis varied considerably between centres, from exclusively PSG to exclusively cardiorespiratory polygraphy. Overall, PSG was used for diagnosis in $33.0 \%$ of all included patients. The proportion of patients investigated with PSG at each participating centre is shown in figure 3. Interestingly, patients investigated by PSG had $\sim 20 \%$ higher AHI when compared with patients investigated by cardiorespiratory polygraphy $\left(29.1 \pm 26.3\right.$ events $\cdot h^{-1}, \mathrm{n}=1,343$, versus $23.2 \pm 23.5$ events $\cdot \mathrm{h}^{-1}, \mathrm{n}=3,245$, respectively; $\mathrm{p}<0.0001$; tables 2 and 3). The mean $\pm \mathrm{SD}$ analysed sleep time in polygraphic recordings was $434 \pm 59.2 \mathrm{~min}$, while the mean \pm SD objectively assessed total sleep time in patients undergoing PSG studies was $385 \pm 81.6 \mathrm{~min}$. The difference in AHI between the two groups could not be explained by differences in sex, age, BMI or selfreported sleepiness (ESS) (data not shown).

Cardiovascular comorbidity was frequent $(49.1 \%$; table 1$)$ among referred patients and included systemic hypertension, ischaemic heart disease and, to a lesser extent, cerebrovascular disease. Other common comorbidities included metabolic disease $(32.9 \%)$, mainly hyperlipidaemia and diabetes (table 1 ). Pulmonary disease was also prevalent $(14.2 \%)$ in the patient cohort. Overall, the patient populations in sleep disorder centres across the ESADA network expressed a high comorbidity both in males and females. Interestingly, cardiovascular comorbidity was present in $48.6 \%$ and $50.4 \%$ of patients investigated by polygraphy and PSG, respectively, suggesting that the sleep diagnostic test procedure was not affected by comorbidity.

The distribution of comorbidities after stratification of patients according to BMI classes $<25,25-<30$ and $\geqslant 30 \mathrm{~kg} \cdot \mathrm{m}^{-2}$ is illustrated in table 4 . As expected, the severity of OSA expressed as AHI increased linearly from $19 \pm 16$ to $25 \pm 19$ and
FIGURE 3. The proportion of patients studied with a polysomnographiy (PSG) sleep study at each centre participating in the European Sleep Apnoea Database (ESADA) study. Med Univ: Medical University; Inst TBC LD: Institute of Tuberculosis and Lung Diseases.

$36 \pm 5$ events $\cdot \mathrm{h}^{-1}$ across the three groups. Similarly, the prevalence of systemic hypertension, all cardiovascular disease, metabolic disease, pulmonary disease as well as ESS score increased with BMI class and was, with the exception of pulmonary disease, consistently higher in patients with OSA within each BMI class.

\section{DISCUSSION}

The ESADA study has been initiated to systematically investigate patient characteristics, diagnostic procedures and treatment outcomes at European sleep disorder centres. This project, which specifically addresses patients referred to a sleep centre with suspected OSA diagnosis, currently involves 22 sleep centres from 16 countries across Europe. The web-based report format has enabled rapid recruitment and the database represents a growing resource for assessment of morbidity, comorbid conditions, medication and health economic evaluation in OSA. Moreover, the database will enable future outcome research, strategic search for biological markers of disease and risk, and genetic studies in sleep apnoea.

The ESADA cohort was started as a result of a collaborative network of researchers in the European COST Action B26 programme recognising that health care allocations for diagnosis and treatment of OSA are unevenly distributed across Europe. Hence, the programme aims to identify improved standard of care and better conformity in sleep medicine practice. The ESADA has also recognised the lack of a large-scale sleep laboratory patient cohort that includes systematically collected information on sleep study data, anthropometrics, prevalence of comorbid conditions and records of ongoing medication. It is believed that such cohorts are needed to enable future genetic and outcome research in OSA. 


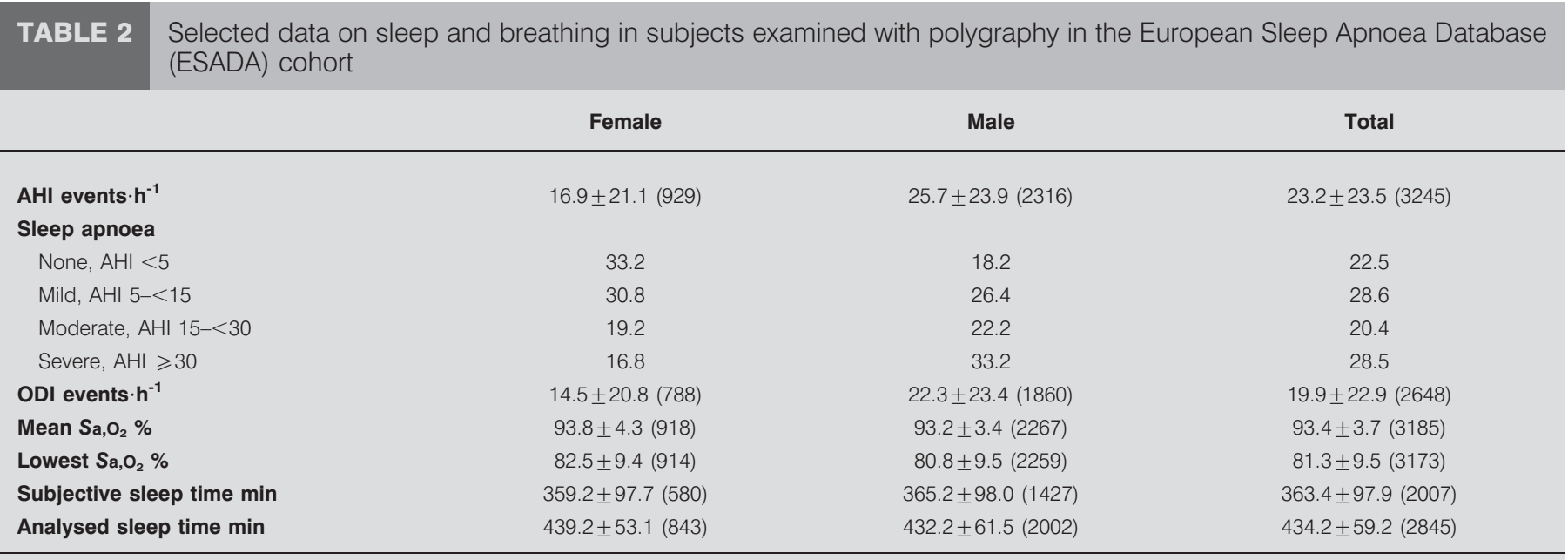

Data are presented as mean \pm SD (n) or \%. AHI: apnoea/hypopnoea index; ODI: oxygen desaturation index; $\mathrm{Sa}_{2} \mathrm{O}_{2}$ : arterial oxygen saturation.

The first data analysis of the ESADA has demonstrated an approximately four-fold difference between centres in terms of reported severe sleep apnoea. This difference appears to reflect considerable variation in referral patterns and possibly variable public and professional awareness of OSA across Europe. The sleep study method, which varied between centres, appeared to influence the reported AHI, as the average severity was $\sim 20 \%$ higher in patients investigated with PSG and not explained by sex, age, BMI or subjective sleepiness. It is likely that the wider definition of hypopnoea, which includes a classification based on arousal, in part accounted for the difference. Another explanation would be that the analysis time (lights off to lights on) includes wake periods during the night leading to a dilution of the reported AHI value as demonstrated by data from the Sleep Heart Health Study [12]. In fact, this interpretation was supported by the $13 \%$ difference between patients with objectively assessed total sleep time by PSG and analysed time in patients undergoing polygraphic recording.

In this study, sleep apnoea severity was higher among males than females, as expected [13], whereas there was no difference between males and females in terms of self-reported measures of sleepiness. This finding appears to support those of another population study that used an AHI cut-off of 15 events $\cdot \mathrm{h}^{-1}$ and reported a similar proportion of males and females in the sleepy and nonsleepy groups [14]. Other observations include the high prevalence of cardiovascular and metabolic disease, which confirms previously published studies [15-17]. Moreover, consistent with several of these studies, both sleep apnoea and obesity appear to contribute to cardiovascular and metabolic morbidity. Taken together, it appears that the ESADA, by representing a sleep laboratory cohort, will recruit proportionally far more severe cases with sleep-disordered breathing than those reported in other large-scale population studies, such as the Sleep Heart Health Study [18] and the Wisconsin Sleep Cohort [1].

The ESADA cohort represents a unique cohort in several aspects. However, the applied recruitment system offers both strengths and weaknesses. The inclusion criteria in the study were adapted to the locally applied clinical routines at each centre in order to visualise differences in medical practice and referral patterns. Data obtained will therefore reflect current practice and will be influenced by geographical differences and differences in healthcare systems. This degree of local

TABLE 3 Sleep study results on patients investigated by polysomnography (PSG)

\begin{tabular}{|c|c|c|c|}
\hline AHI events $\cdot h^{-1}$ & $22.3 \pm 25.0(365)$ & $31.7 \pm 26.3(978)$ & $29.1 \pm 26.3(1343)$ \\
\hline None, $\mathrm{AHI}<5$ & 26.0 & 12.2 & 15.9 \\
\hline Mild, AHI 5-15 & 28.2 & 21.1 & 23.9 \\
\hline Moderate, $\mathrm{AHI}>15-30$ & 20.9 & 23.1 & 22.4 \\
\hline Mean $\mathrm{Sa}, \mathrm{O}_{2} \%$ & $93.6 \pm 3.7(428)$ & $93.1 \pm 4.6(1154)$ & $93.2 \pm 4.4(1582)$ \\
\hline Lowest $\mathrm{Sa}, \mathrm{O}_{2} \%$ & $83.7 \pm 10.2(428)$ & $81.2 \pm 11.1(1149)$ & $81.9 \pm 11.3(1577)$ \\
\hline Total sleep time by PSG min & $385.1 \pm 82.7(428)$ & $384.9 \pm 81.3(1151)$ & $385.0 \pm 81.6(1579)$ \\
\hline Subjective sleep time by questionnaire min & $402.6 \pm 91.5(230)$ & $396.6 \pm 91.4(690)$ & $398.1 \pm 91.4(920)$ \\
\hline
\end{tabular}

Data are presented as mean \pm SD (n) or \%. AHI: apnoea/hypopnoea index; ODI: oxygen desaturation index; $\mathrm{Sa}_{2} \mathrm{O}_{2}$ : arterial oxygen saturation. 


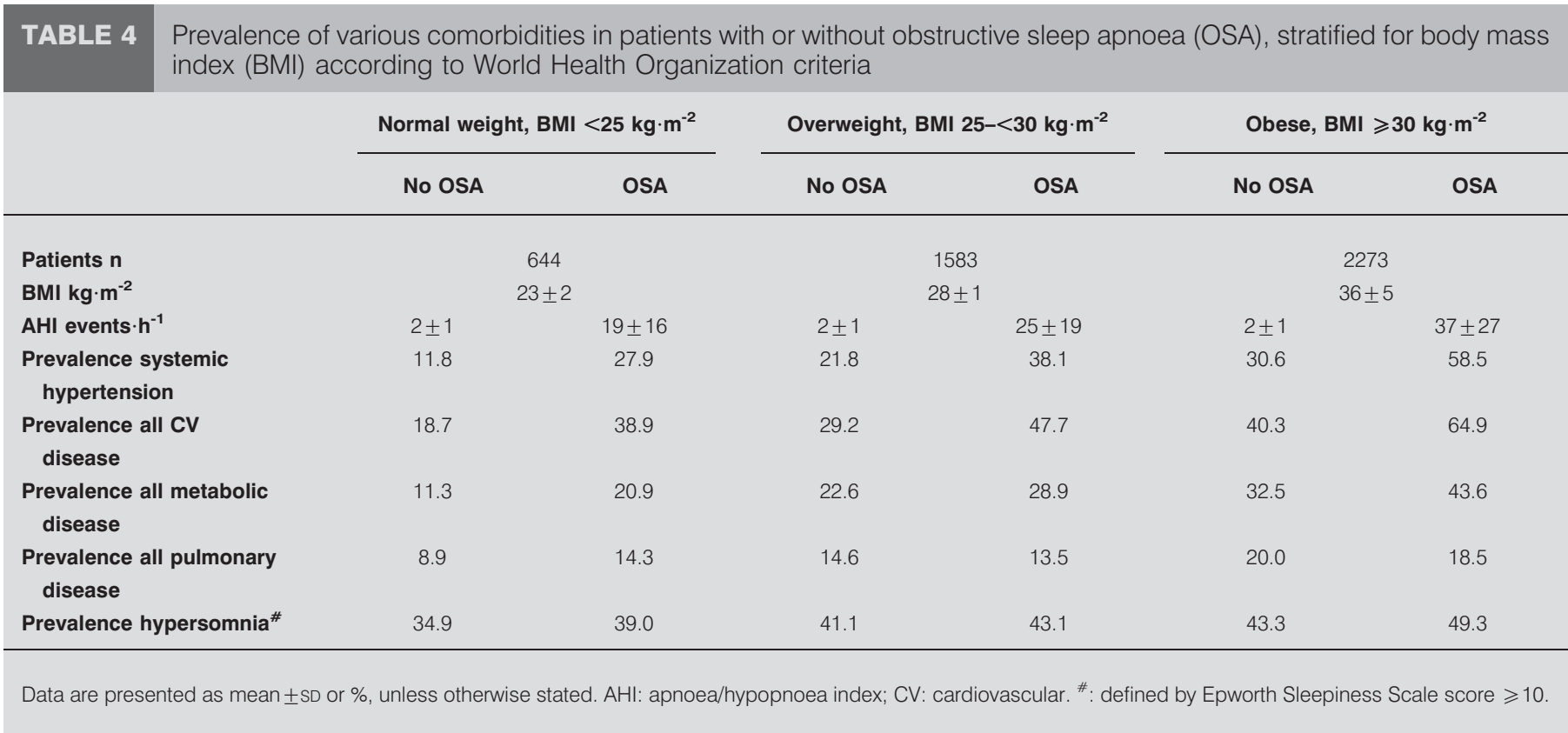

conformity has enabled an acceleration of patient recruitment, with more than 5,000 patients included in the study during the first 28 months. One additional facilitating factor is the webbased report format, which guarantees conformity of reported data sets. Instructions were given to avoid preselection bias during recruitment and centres were asked to apply randomness in the inclusion of new patients into the cohort. Recruited patients will therefore reflect those produced by the local referral patterns. Data on comorbid conditions and medications will reflect the characteristics of patients referred with suspected OSA across European centres. Similarly, routines for treatment will vary between centres, as will the applied standards for follow-up of treated patients. The protocol is not designed to change any currently applied routines and the database will therefore contain patients with various forms of treatment investigated at different time-points after initiation of treatment.

Some methodological weaknesses of the ESADA cohort represent compromises to the recruitment environment. For instance, the nonstandardisation of sleep test equipment may be seen as a weakness that may lead to a reduced sharpness of OSA severity classification. However, by accounting for type of diagnostic equipment used, a future prospective analysis will provide a unique possibility to evaluate if the recording technique used has an influence on patient outcome. Blood samples for risk factor classification are obtained in all participating patients but the timing of sampling has not been standardised. Hence, samples may be affected by diurnal variation and, in some cases, nonfasting conditions. Information on comorbidity is obtained by different methods, such as hospital registry data, subjective report from the patient or by information obtained from the referral notes. Admittedly this may lead to incomplete information and classification. Only $14.3 \%$ of this sleep laboratory cohort is of normal weight $\left(\leqslant 25 \mathrm{~kg} \cdot \mathrm{m}^{-2}\right)$. Thus, analyses of comorbidities in normalweight OSA may be less powerful than in overweight OSA.
Clinical investigations for classification of end-organ damage, e.g. by echocardiography, radiological investigation or electrocardiography, are not systematically performed for evident reasons. Hence, conditions such as left ventricular hypertrophy or cardiac arrhythmia may be consistently underreported as no objective data is obtained. It is also possible that additional resources should be focused at improved phenotypic characterisation of sleep apnoea cases. This may be particularly interesting considering that blood samples for genetic analysis are obtained in the study. Phenotypic information, such as facial structure and upper airway structural measures using various imaging techniques, were considered too complex for general application in the protocol but the continued recruitment into the database may enable future sampling of this type of data in one or several participating centres.

In summary, the ESADA study represents a large-scale multicentre collaborative approach to rapidly recruit one of the world's largest ongoing observational cohorts of patients with sleep-disordered breathing. This first cross-sectional analysis has confirmed a high prevalence of cardiovascular and metabolic morbidity in referred patients. The cohort is expected to enable a unique opportunity to perform in-depth analyses of local referral patterns and to define occurrence of comorbid conditions in patients seen at different European sleep centres. Other opportunities include large-scale outcome research based on genotyping and potentially identification of biomarkers for sleep-disordered breathing.

\section{SUPPORT STATEMENT}

The ESADA network has received support from the European Union Cooperation in Science and Technology (COST) Action B26 programme.

\section{STATEMENT OF INTEREST}

Statements of interest for M. Bonsignore and for the study itself can be found at www.erj.ersjournals.com/site/misc/statements.xhtml 


\section{ACKNOWLEDGEMENTS}

The contribution of recruiting physicians and all technical support staff at the participating centres is gratefully acknowledged. Excellent technical assistance in terms of centre monitoring and database maintenance has been provided by D. Zou, A-C. Lundquist and J. Norum (Sleep Laboratory, Sahlgrenska University Hospital, Gothenburg, Sweden).

The author affiliations are as follows. J. Hedner and L. Grote: Sleep Laboratory, Sahlgrenska University Hospital. M. Bonsignore and O. Marrone: CNR Institute of Biomedicine and Molecular Immunology, Palermo, Italy. W. McNicholas: Dept of Respiratory Medicine, St Vincent's University Hospital, Dublin, Ireland. P. Lavie: Technion Sleep Laboratory, Rambam Medical Centre, Haifa, Israel. G. Parati and C. Lombardi: Instituto Auxologico Italiano, Ospedale San Luca, Milan, Italy. P. Sliwinski: Institute of Tuberculosis and Lung Diseases, Dept of Diagnosis and Treatment of Respiratory Failure, Warsaw, Poland. F. Barbé: Servei Pneumologia Hospital Arnau de Vilanova and Hospital Santa Maria, Lleida, and CIBERES, Madrid, Spain. W. DeBacker and H. Vrints: Dept of Pulmonary Medicine, University Hospital Antwerp, Edegem-Antwerp, Belgium. P. Escourrou: Service d'Éxplorations Fonctionnelles Multidisciplinaires, Hospital Antoine Beclere, Clamart, France. I. Fietze and T. Penzel: Schlafmedizinisches Zentrum, Berlin, Germany. J.A. Kvamme: Sleep Laboratory, ENT Dept, Førde Central Hospital, Førde, Norway. J.F. Masa: Hospital San Pedro de Alcàntara, Cáceres, CIBERES, Madrid, Spain. J.M. Montserrat: Hospital Clinic i Provincial de Barcelona, Barcelona, IDIBAPS Barcelona, and CIBERES, Madrid, Spain. M. Pretl: Sleep Disorders Centre, Dept of Neurology, First Faculty of Medicine, Charles University, Prague, Czech Republic. R. Riha: Dept of Sleep Medicine, Royal Infirmary Edinburgh, Edinburgh, UK. D. Rodenstein: Cliniques Universitaires, Brussels, Belgium. T. Saaresranta: Turku University Central Hospital Paimio, Hospital Dept of Pulmonary Diseases, Preitilä, Finland. R. Schulz: Sleep Disorders Centre, University of Giessen, Lung Centre, Giessen, Germany. R. Tkacova: Dept of Respiratory Medicine and Tuberculosis, Faculty of Medicine, P.J. Safarik University and L. Pasteur University Hospital, Kosice, Slovakia. G. Varoneckas: Institute Psychophysiology and Rehabilitation, Palanga, Lithuania. A. Vitols: Institute of Cardiology, University of Latvia, Riga, Latvia. J. Zielinski: Dept of Pneumonology, Warsaw Medical University, Warsaw, Poland.

\section{REFERENCES}

1 Young T, Palta M, Dempsey J, et al. The occurrence of sleepdisordered breathing among middle-aged adults. $N$ Engl J Med 1993; 328: 1230-1235.

2 Jennum P, Riha RL. Epidemiology of sleep apnoea/hypopnoea syndrome and sleep-disordered breathing. Eur Respir J 2009; 33: 907-914.
3 Nowak M, Kornhuber J, Meyrer R. Daytime impairment and neurodegeneration in OSAS. Sleep 2006; 29: 1521-1530.

4 Rodenstein D. Driving in Europe: the need of a common policy for drivers with obstructive sleep apnoea syndrome. J Sleep Res 2008; 17: 281-284.

5 McNicholas WT, Bonsignore MR. Sleep apnoea as an independent risk factor for cardiovascular disease: current evidence, basic mechanisms and research priorities. Eur Respir J 2007; 29: 156-178.

6 Pack AI, Gislason T. Obstructive sleep apnea and cardiovascular disease: a perspective and future directions. Prog Cardiovasc Dis 2009; 51: 434-451.

7 Kato M, Adachi T, Koshino Y, et al. Obstructive sleep apnea and cardiovascular disease. Circ J 2009; 73: 1363-1370.

8 WHO Collaborating Centre for Drug Statistics Methodology, Guidelines for ATC Classification and DDD Assignment. 6th Edn. Oslo, World Health Organization, 2003.

9 Ferber R, Millman R, Coppola M, et al. Portable recording in the assessment of obstructive sleep apnea. ASDA standards of practice. Sleep 1994; 17: 378-392.

10 Iber C, Ancoli-Israel S, Chesson A, et al. The AASM Manual for the Scoring of Sleep and Associated Events: Rules, Terminology and Technical Specifications. 1st Edn. Westchester, American Academy of Sleep Medicine, 2007.

11 Johns MW. A new method for measuring daytime sleepiness: the Epworth sleepiness scale. Sleep 1991; 14: 540-545.

12 Silva GE, Goodwin JL, Sherrill DL, et al. Relationship between reported and measured sleep times: the sleep heart health study (SHHS). J Clin Sleep Med 2007; 3: 622-630.

13 Young T, Peppard PE, Gottlieb DJ. Epidemiology of obstructive sleep apnea: a population health perspective. Am J Respir Crit Care Med 2002; 165: 1217-1239.

14 Kapur VK, Baldwin CM, Resnick HE, et al. Sleepiness in patients with moderate to severe sleep-disordered breathing. Sleep 2005; 28 : 472-477.

15 Peker Y, Hedner J, Kraiczi H, et al. Respiratory disturbance index: an independent predictor of mortality in coronary artery disease. Am J Respir Crit Care Med 2000; 162: 81-86.

16 Lavie P, Herer P, Hoffstein V. Obstructive sleep apnoea syndrome as a risk factor for hypertension: population study. BMJ 2000; 320: 479-482.

17 Kiely JL, McNicholas WT. Cardiovascular risk factors in patients with obstructive sleep apnoea syndrome. Eur Respir J 2000; 16: $128-133$.

18 Nieto FJ, Young TB, Lind BK, et al. Association of sleep-disordered breathing, sleep apnea, and hypertension in a large communitybased study. Sleep Heart Health Study. JAMA 2000; 283: 1829-1836. 